Research

\title{
In-vitro antimycobacterial drug susceptibility testing of non-tubercular mycobacteria by tetrazolium microplate assay Manimuthu Mani Sankar ${ }^{1}$, Krishnamoorthy Gopinath ${ }^{1}$, Roopak Singla ${ }^{2}$ and Sarman Singh*1
}

Address: ${ }^{1}$ Clinical Microbiology Division, Department of Laboratory Medicine, All India Institute of Medical Sciences, New Delhi, India and 2Division of Respiratory Medicine, LRS Institute of Tuberculosis and Lung Diseases, New Delhi, India

Email: Manimuthu Mani Sankar - sankar.mm@gmail.com; Krishnamoorthy Gopinath - kgnath@gmail.com; Roopak Singla - rupaks@lrs.nic.in; Sarman Singh* - sarman_singh@yahoo.com

* Corresponding author

Published: II July 2008

Annals of Clinical Microbiology and Antimicrobials 2008, 7:15 doi:10.1 I86/1476-07II-715

This article is available from: http://www.ann-clinmicrob.com/content/7/I/I5

(c) 2008 Sankar et al; licensee BioMed Central Ltd.

This is an Open Access article distributed under the terms of the Creative Commons Attribution License (http://creativecommons.org/licenses/by/2.0), which permits unrestricted use, distribution, and reproduction in any medium, provided the original work is properly cited.

\begin{abstract}
Background: Non-tubercular mycobacteria (NTM) has not been given due attention till the recent epidemic of HIV. This has led to increasing interest of health care workers in their biology, epidemiology and drug resistance. However, timely detection and drug susceptibility profiling of NTM isolates are always difficult in resource poor settings like India. Furthermore, no standardized methodology or guidelines are available to reproduce the results with clinical concordance.
\end{abstract}

Objective: To find an alternative and rapid method for performing the drug susceptibility assay in a resource limited settings like India, we intended to evaluate the utility of Tetrazolium microplate assay (TEMA) in comparison with proportion method for reporting the drug resistance in clinical isolates of NTM.

Methods: A total of fifty-five NTM isolates were tested for susceptibility against Streptomycin, Rifampicin, Ethambutol, Ciprofloxacin, Ofloxacin, Azithromycin, and Clarithromycin by TEMA and the results were compared with agar proportion method (APM).

Results: Of the 55 isolates, 23 (4I.8\%) were sensitive to all the drugs and the remaining 32 (58.2\%) were resistant to at least one drug. TEMA had very good concordance with APM except with minor discrepancies. Susceptibility results were obtained in the median of 5 to 9 days by TEMA. The NTM isolates were highly sensitive against Ofloxacin ( $98.18 \%$ sensitive) and Ciprofloxacin ( $90.09 \%$ sensitive). M. mucogenicum was sensitive only to Clarithromycin and resistant to all the other drugs tested. The concordance between TEMA and APM ranged between $96.4-100 \%$.

Conclusion: Tetrazolium Microplate Assay is a rapid and highly reproducible method. However, it must be performed only in tertiary level Mycobacteriology laboratories with proper bio-safety conditions.

\section{Background}

The number and species of Non-tubercular mycobacteria
(NTM) isolated from clinical specimens is continuously increasing with the advancement of microbiological 
detection methods. Numbers of reports have confirmed the pathogenic role, morbidity and mortality caused by NTMs in AIDS patients [1-4]. The infection due to NTM is difficult to treat because of their intrinsic resistance to the major classes of drugs, probably due to their habitat $[5,6]$. Therefore to choose an effective therapeutic drug, anti mycobacterial susceptibility testing becomes a primary step for the management of the NTM disease. Though there are specific recommendations and guidelines prescribed by the World Health Organization (WHO) and Centre for Disease Control (CDC) regarding anti-tubercular drug susceptibility methods for Mycobacterium tuberculosis there are no such guidelines for NTMs. As routine drug susceptibility testing of NTMs are discouraged, there are circumstances where susceptibility testing is warranted [7].

Traditionally drug susceptibility testing in mycobacterial isolates is performed in agar and Lowenstein Jensen (LJ) medium which is considered as a 'gold standard' but it is cumbersome and available only in few reference laboratories in a country like India where tuberculosis is highly endemic. Even though automated systems dramatically reduced the time for detecting drug resistance in mycobacterial isolates but these are more expensive and not feasible especially in a low resource setting [8-12].

Since there was a need for rapid, easy to perform and qualitative method for predicting the Minimal Inhibitory Concentration (MICs) for NTM isolates, Mshana et al [13] developed a rapid colorimetric method employing oxidation-reduction indicator Tetrazolium bromide to perform drug susceptibility testing for Mycobacterium tuberculosis which was found to be promising and cost effective [14].
Therefore, we intended to assess the performance and efficiency of Tetrazolium Microplate Assay (TEMA) for determining MICs of Streptomycin (STR), Rifampicin (RIF), Ethambutol (ETH), Ciprofloxacin (CIP), Ofloxacin (OFL), Azithromycin (ATH) and Clarithromycin (CLA) against clinically isolated NTMs. The results obtained by TEMA were compared with Agar Proportion Method (APM) on Middle brook 7H10 agar plates.

\section{Methods \\ Mycobacterial isolates}

A total of fifty-five NTMs isolated from patients referred to our laboratory for mycobacterial isolation were included, as shown in Table 1. These strains were identified and confirmed as NTM by biochemical methods such as heat stable catalase, niacin and nitrate production, aryl sulphatase and sodium chloride tolerance test [15] and DNA sequencing was done with targets of 16S rRNA and 16S23S Internal Transcribed Spacer sequences or $h s p 65$ as previously reported elsewhere [16]. Standard strains [M. avium (NCTC-8551), M. chelonae (TMC-1544), M. xenopi (NCTC-10042), M. phlei (NCTC-8156), M. intracellularae (TMC1406), M. simiae (TMC 1226), M. fortuitum (TMC1529), M. smegmatis (TMC1546), M. terrae (TMC1450) \& M. kansasii] used as controls against the clinical NTM isolates were kindly gifted by Dr. V. M. Katoch, National JALMA Institute of Leprosy and Other Mycobacterial Diseases, Agra, India.

\section{Inoculum and drug preparation}

Antimycobacterial drugs were procured from commercial source (Sigma ${ }^{\circledast}$, U.S.A) and solubilised according to the manufacturer's recommendations in appropriate solvents and sterilized by filtering through $0.22 \mu \mathrm{m}$ membrane fil-

Table I: Mean MICs of drugs $(\mu \mathrm{g} / \mathrm{mL})$ against clinical isolates of Non-tubercular mycobacterial isolates

\begin{tabular}{|c|c|c|c|c|c|c|c|c|c|c|c|c|c|c|}
\hline \multirow[t]{2}{*}{ Clinical isolate (n) } & \multicolumn{2}{|c|}{ STR } & \multicolumn{2}{|c|}{ RIF } & \multicolumn{2}{|c|}{ ETH } & \multicolumn{2}{|c|}{ CIP } & \multicolumn{2}{|c|}{ OFL } & \multicolumn{2}{|c|}{ ATH } & \multicolumn{2}{|c|}{ CLA } \\
\hline & $\mathbf{M}$ & SD & $\mathbf{M}$ & SD & $\mathbf{M}$ & SD & $\mathbf{M}$ & SD & $\mathbf{M}$ & SD & M & SD & $\mathbf{M}$ & SD \\
\hline M. avium (16) & 0.6 & 1.69 & 0.5 & 0.96 & 57.7 & 66.63 & 0.27 & 0.48 & 0.6 & 0.16 & 158.5 & 215.9 & 22.2 & 32.53 \\
\hline M. abscess (I) & 0.22 & - & 0.22 & - & 138.3 & - & 0.13 & - & 0.66 & - & 1.37 & - & 0.16 & - \\
\hline M. chelonae (7) & 3.98 & 10.49 & 1.03 & 2.58 & 19.98 & 52.17 & 0.13 & - & 0.66 & - & 1.95 & 1.55 & 0.23 & 0.18 \\
\hline M. fortuitum (7) & 0.05 & - & 0.29 & 0.63 & 19.98 & 52.17 & 0.13 & - & 0.66 & - & 36.8 & 93.97 & 12.02 & 31.38 \\
\hline M. intracellulare (I) & 0.54 & - & 0.54 & - & 0.27 & - & 0.13 & - & 0.66 & - & 1.37 & - & 0.16 & - \\
\hline M. kansasii (I) & 0.21 & - & 0.05 & - & 0.27 & - & 0.13 & - & 0.66 & - & 1.37 & - & 0.32 & - \\
\hline M. mucogenicum (I) & 0.86 & - & 27.6 & - & 138.3 & - & 0.53 & - & 2.06 & - & 43.75 & - & 1.3 & - \\
\hline M. parascrofulaceum (I) & 1.72 & - & 0.05 & - & 138.3 & - & 0.13 & - & 0.66 & - & 1.37 & - & 0.16 & - \\
\hline M. phlei (3) & 0.12 & 0.08 & 0.12 & 0.08 & 49.07 & 77.38 & 0.77 & 1.11 & 0.66 & - & 59.24 & 100.24 & 27.84 & 47.94 \\
\hline M. scrofulaceum (I) & 0.21 & - & 0.05 & - & 0.27 & - & 0.13 & - & 0.66 & - & 1.37 & - & 0.32 & - \\
\hline M. simiae (4) & 0.47 & 0.83 & 0.92 & 1.68 & 69.28 & 79.69 & 4.26 & 8.16 & 0.66 & - & 1.37 & - & 0.16 & - \\
\hline M. smegmatis (3) & 0.11 & 0.093 & 0.54 & - & 46.28 & 79.69 & 0.13 & - & 0.66 & - & 30.07 & 49.72 & 7.04 & 11.91 \\
\hline M. terrae (7) & 1.81 & 2.57 & 1.87 & 2.54 & 20.37 & 52.01 & 0.26 & 0.33 & 0.6 & 0.14 & 1.37 & - & 12.02 & 31.38 \\
\hline M. xenopi (2) & 0.05 & - & 0.05 & - & 0.27 & - & 0.13 & - & 0.66 & - & 1.37 & - & 0.16 & - \\
\hline
\end{tabular}

STR: Streptomycin, RIF: Rifampicin, ETH: Ethambutol, CIP: Ciprofloxacin, OFL: Ofloxacin, ATH: Azithromycin, CLA: Clarithromycin, M: Mean, SD: Standard deviation. 
ter (Millipore ${ }^{\circledast}$, Ireland). Tetrazolium bromide [3-(4, 5dimethylthiazol-2-yl)-2,5-diphenyl-tetrazolium bromide] (Sigma ${ }^{\circledR}$, USA.) was prepared at a concentration of $1 \mathrm{mg} / \mathrm{ml}$ in absolute ethanol and $1.5 \mathrm{ml}$ of $10 \%$ Tween 80 and the stock was stored in dark at $4{ }^{\circ} \mathrm{C}$. The mycobacterial inoculum was prepared from log-phase culture of the NTMs on LJ slants and their turbidity was adjusted to McFarland Standard No. 1.

\section{Tetrazolium Microplate Assay}

TEMA was performed as previously described [13] with minor modifications (Illustrated in Fig. 1). Briefly, $100 \mu \mathrm{L}$ of Middlebrook 7H9 broth (pH 7.2; Sigma ${ }^{\circledR}$, USA) was added to columns 2 to 11 in rows $A$ to $G$ (labeled on microtitre plates). One-hundred-microliters of $2 \times$ concentration of drug were added to columns 1 and 2 . The antibiotics were serially diluted twofold in consecutive columns by transferring $100 \mu \mathrm{L}$, except for column 10 , where $100 \mu \mathrm{L}$ of excess medium was discarded. The final drug concentrations in the wells were set as follows: STR: 27.6 to $0.0539 \mu \mathrm{g} / \mathrm{mL}$, RIF: 27.6 to $0.0539 \mu \mathrm{g} / \mathrm{mL}$, ETH: 138.3 to $0.27 \mu \mathrm{g} / \mathrm{mL}, \mathrm{CIP}: 66$ to $0.132 \mu \mathrm{g} / \mathrm{mL}$, OFL: 33 to $0.66 \mu \mathrm{g} / \mathrm{mL}$, ATH: 500 to $1.367 \mu \mathrm{g} / \mathrm{mL}$ and CLA 83.2 to $0.162 \mu \mathrm{g} / \mathrm{mL}$. Hundred microliters of mycobacterial suspension (set to McFarland Standard No.1) was added to wells in rows A to $\mathrm{G}$ in columns 1 to 11 . The wells in column 11 served as inoculum-growth control with no drugs. The plates were incubated at $37^{\circ} \mathrm{C}$ for 5 days. On day $5,50 \mu$ l of the tetrazolium dye was added to well A1 1 and the plate was then incubated at $37^{\circ} \mathrm{C}$ for $24 \mathrm{~h}$. A change in colour from yellow to purple indicated growth of bacteria and the MICs was interpreted visually.

\section{Agar Proportion method}

APM method was performed on Middlebrook 7H10 agar plates as previously described by others $[15,17]$. The drug concentrations in the agar plates were STR $8 \mu \mathrm{g} / \mathrm{mL}$, RIF 2 $\mu \mathrm{g} / \mathrm{mL}$, ETH $40 \mu \mathrm{g} / \mathrm{mL}$, CIP $2 \mu \mathrm{g} / \mathrm{mL}$, OFL $2 \mu \mathrm{g} / \mathrm{mL}$, ATH $200 \mu \mathrm{g} / \mathrm{mL}$, and CLA $80 \mu \mathrm{g} / \mathrm{mL}$. The strains were classified as susceptible to a drug if the number of colonies was $<1 \%$ and resistant if the number of colonies was $>10 \%$ in the drug containing media to the number of colonies on the control plate without any drug.

\section{Data analysis}

Analysis of data obtained by TEMA and the APM was carried by SPSS ${ }^{\circledast}$ software (version 11.5). Receiver Operating Characteristic (ROC) curve analysis was performed to measure the Area Under Curve (AUC).

\section{Results}

The sensitivity and resistance patterns against STR, RIF, ETH, CIP, OFL, ATH and CLA by both TEMA and APM are described in Table 1. All the standard strains of NTMs were sensitive to all the tested drugs. The mean MICs values of the drugs (Table 2) tested by TEMA varied between

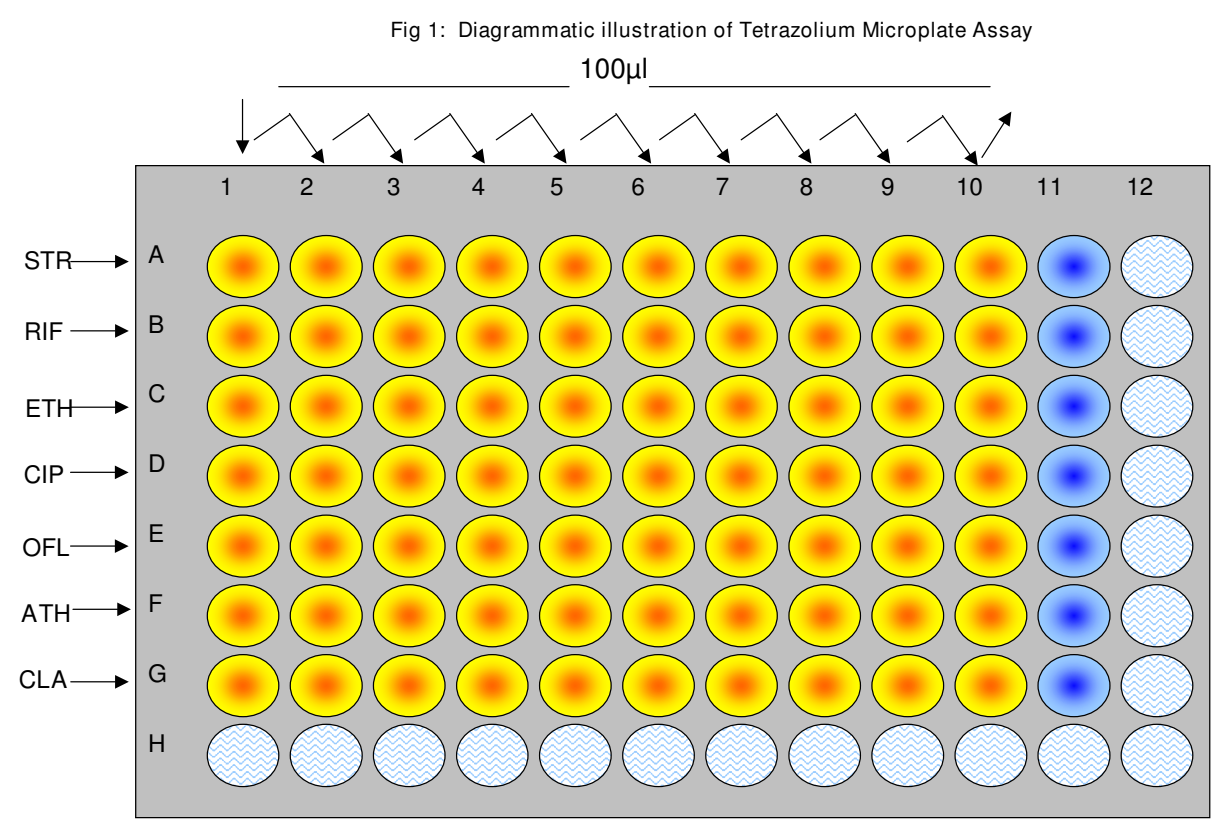

STR: Streptomycin, RIF: Rifampicin, ETH: Ethambutol, CIP: Ciprofloxacin, OFL: Ofloxacin, ATH: Azithromycin, CLA: Clarithromycin,: $\bigcirc$ Growth Control,: $\bigcirc$ Distilled Water.

\section{Figure I}

Diagrammatic illustration of tetrazolium microplate assay. 
Table 2: Comparison of TEMA and APM for antimycobacterial susceptibility testing of Non-tubercular mycobacterial isolates

\begin{tabular}{|c|c|c|c|c|c|c|c|c|c|c|c|c|c|c|}
\hline \multirow[b]{3}{*}{ TEMA } & \multicolumn{14}{|c|}{ APM } \\
\hline & \multicolumn{2}{|c|}{ STR } & \multicolumn{2}{|c|}{ RIF } & \multicolumn{2}{|c|}{ ETH } & \multicolumn{2}{|c|}{ CIP } & \multicolumn{2}{|c|}{ OFL } & \multicolumn{2}{|c|}{ ATH } & \multicolumn{2}{|c|}{ CLA } \\
\hline & $\mathbf{S}$ & $\mathbf{R}$ & $\mathbf{S}$ & $\mathbf{R}$ & $\mathbf{S}$ & $\mathbf{R}$ & $\mathbf{S}$ & $\mathbf{R}$ & $\mathbf{S}$ & $\mathbf{R}$ & $\mathbf{S}$ & $\mathbf{R}$ & $\mathbf{S}$ & $\mathbf{R}$ \\
\hline Sensitive & 43 & 1 & 45 & 0 & 36 & 0 & 50 & 0 & 53 & 0 & 43 & 0 & 45 & 0 \\
\hline Resistant & 1 & 10 & I & 9 & 0 & 19 & 0 & 5 & 1 & 1 & 1 & 11 & 1 & 9 \\
\hline Total & 44 & II & 46 & 9 & 36 & 19 & 50 & 5 & 54 & 1 & 44 & 11 & 46 & 9 \\
\hline Percentage & $(80 \%)$ & $(20 \%)$ & $(83.6 \%)$ & (16.4\%) & $(65.5 \%)$ & $(34.5 \%)$ & (91\%) & (9\%) & $(98.2 \%)$ & $(1.8 \%)$ & $(80 \%)$ & $(20 \%)$ & $(83.6 \%)$ & $(16.4 \%)$ \\
\hline Concordance & \multicolumn{2}{|c|}{$96.4 \%$} & \multicolumn{2}{|c|}{$98 \%$} & \multicolumn{2}{|c|}{$100 \%$} & \multicolumn{2}{|c|}{$100 \%$} & \multicolumn{2}{|c|}{$98 \%$} & \multicolumn{2}{|c|}{$98 \%$} & \multicolumn{2}{|c|}{$98 \%$} \\
\hline
\end{tabular}

TEMA: Tetrazolium Microplate Assay, STR: Streptomycin, RIF: Rifampicin, ETH: Ethambutol, CIP: Ciprofloxacin, OFL: Ofloxacin, ATH: Azithromycin, CLA: Clarithromycin, S: Sensitive, R: Resistant.

clinical isolates belonging to different species. Of the 55 isolates, $23(41.8 \%)$ were sensitive to all the drugs and the remaining $32(58.2 \%)$ were resistant to at least one drug (Fig. 2).

When the efficiency of TEMA and APM were compared for detecting susceptibility patterns in NTM isolates, they showed 96.4\% concordance for STR (Fig. 3), 98\% con- cordance for RIF (Fig. 4), 100\% for ETH (Fig. 5), and CIP (Fig. 6), and 98\% for OFL (Fig. 7), ATH (Fig. 8) and CLA (Fig. 9). The Area Under Curve (AUC) by ROC curve analysis for STR was 0.943, RIF was 0.950, ETH was 1.000, CIP was 1.000 , OFL was 0.750 , ATH was 0.958 and CLA was 0.950 . NTM isolates showed $90.09 \%$ and $98.18 \%$ of susceptibility to Ciprofloxacin and Ofloxacin, respectively. TEMA was rapid and susceptibility results could be

Fig 2: Drug resistance patterns of NTM

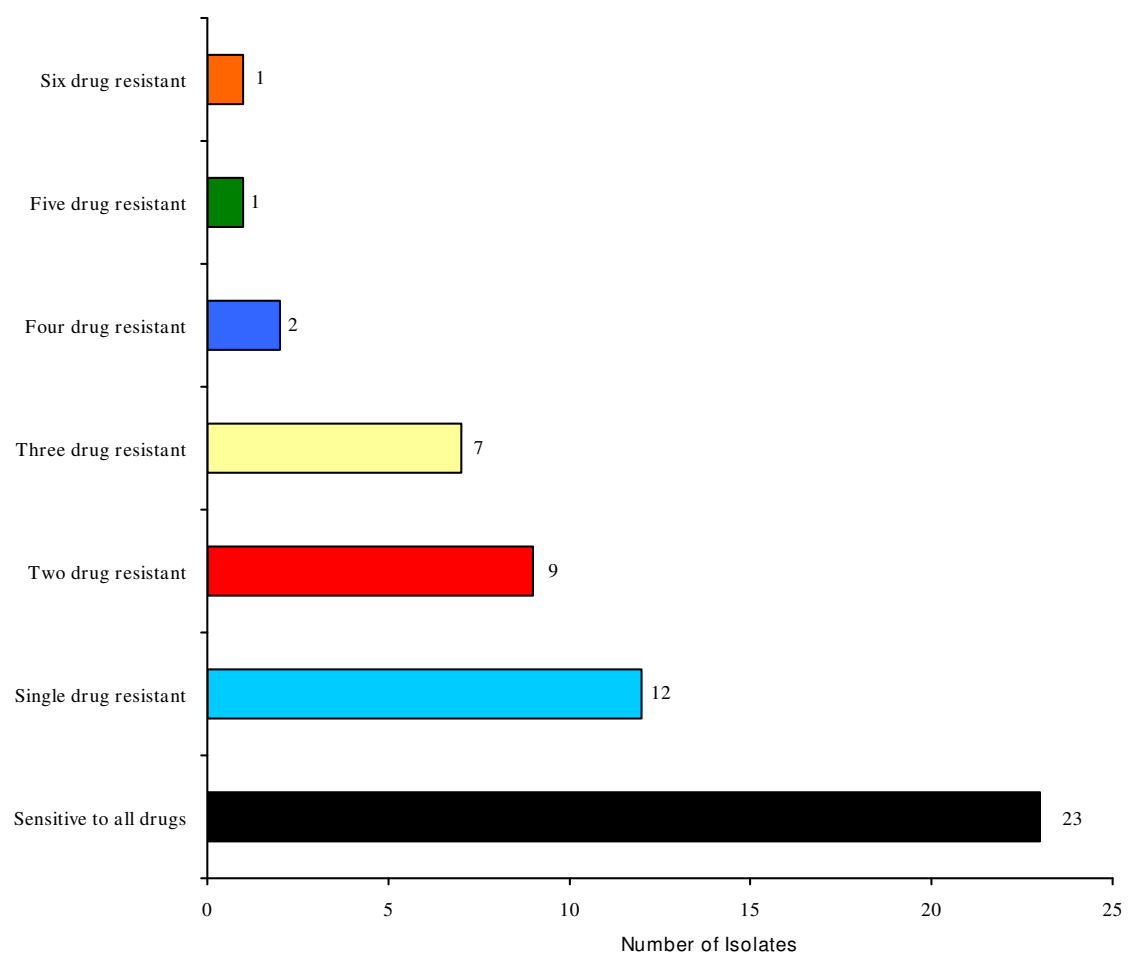

Figure 2

Drug resistance patterns of NTM. 


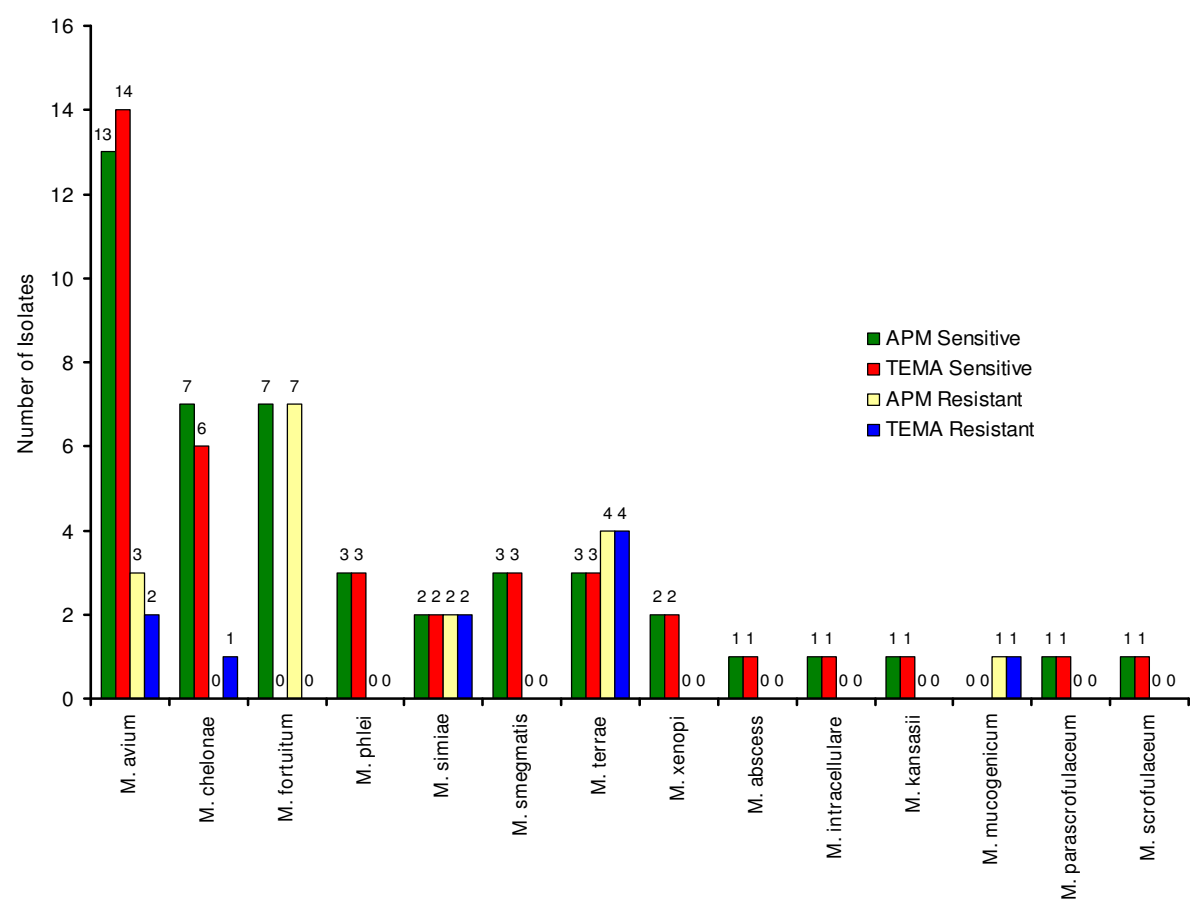

Figure 3

Drug resistance patterns in individual species of NTM against Streptomycin.

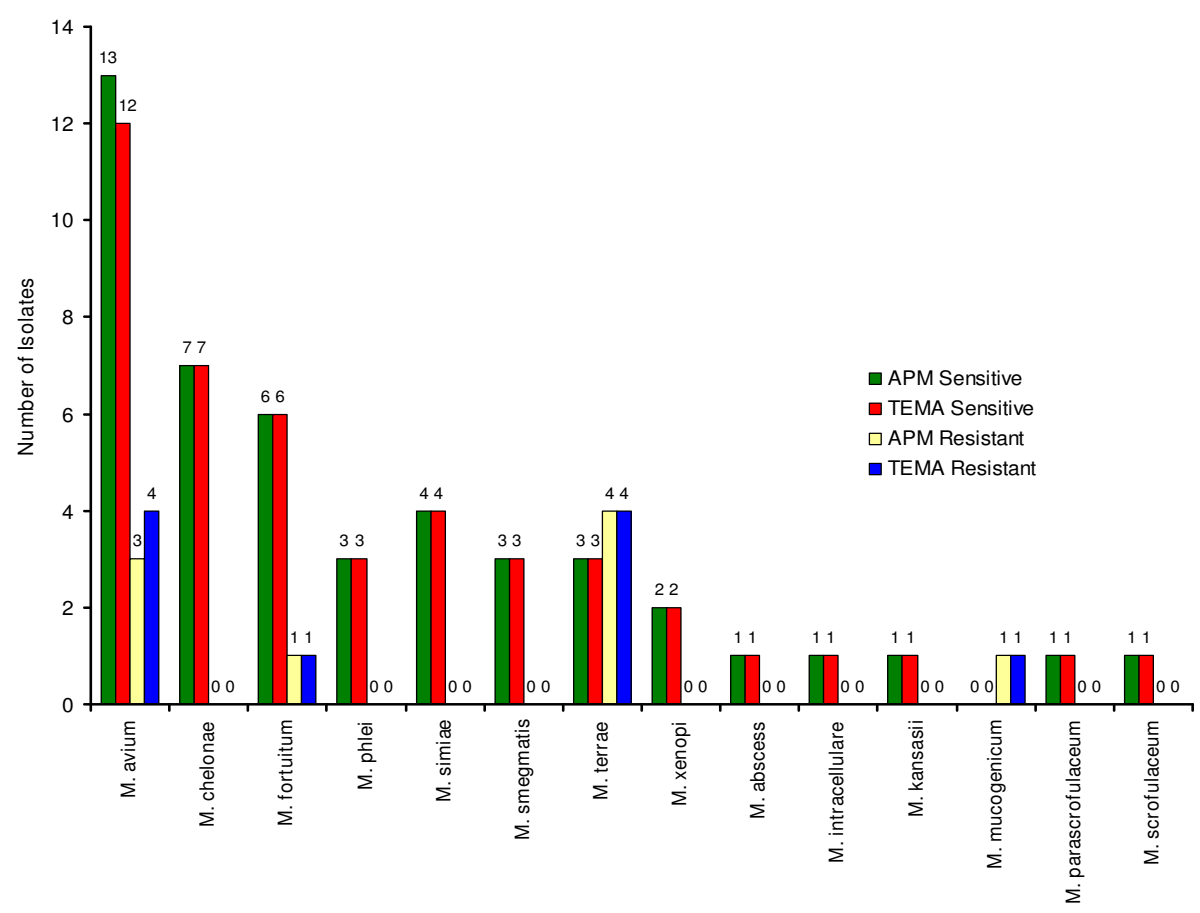

Figure 4

Drug resistance patterns in individual species of NTM against Rifampicin. 


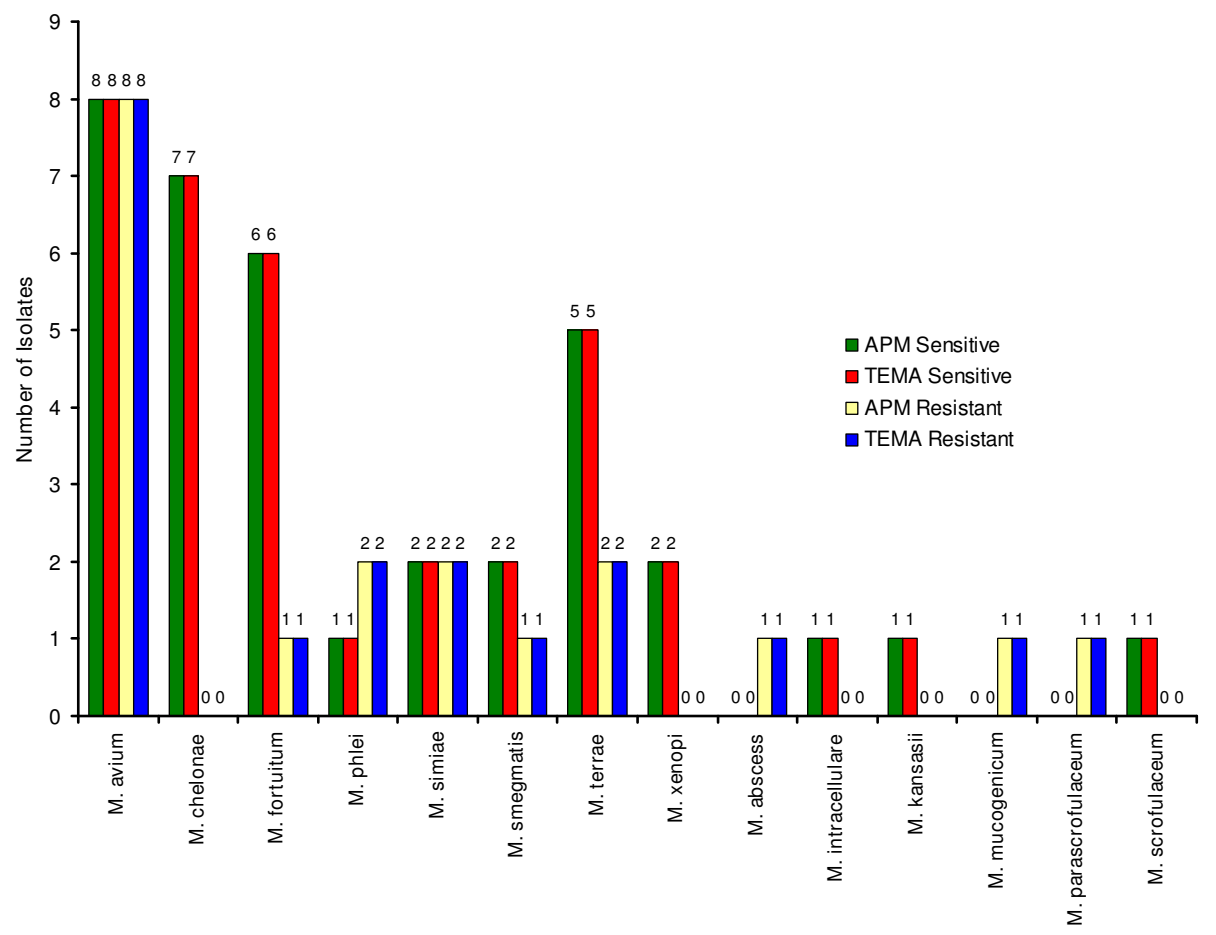

\section{Figure 5}

Drug resistance patterns in individual species of NTM against Ethambutol.

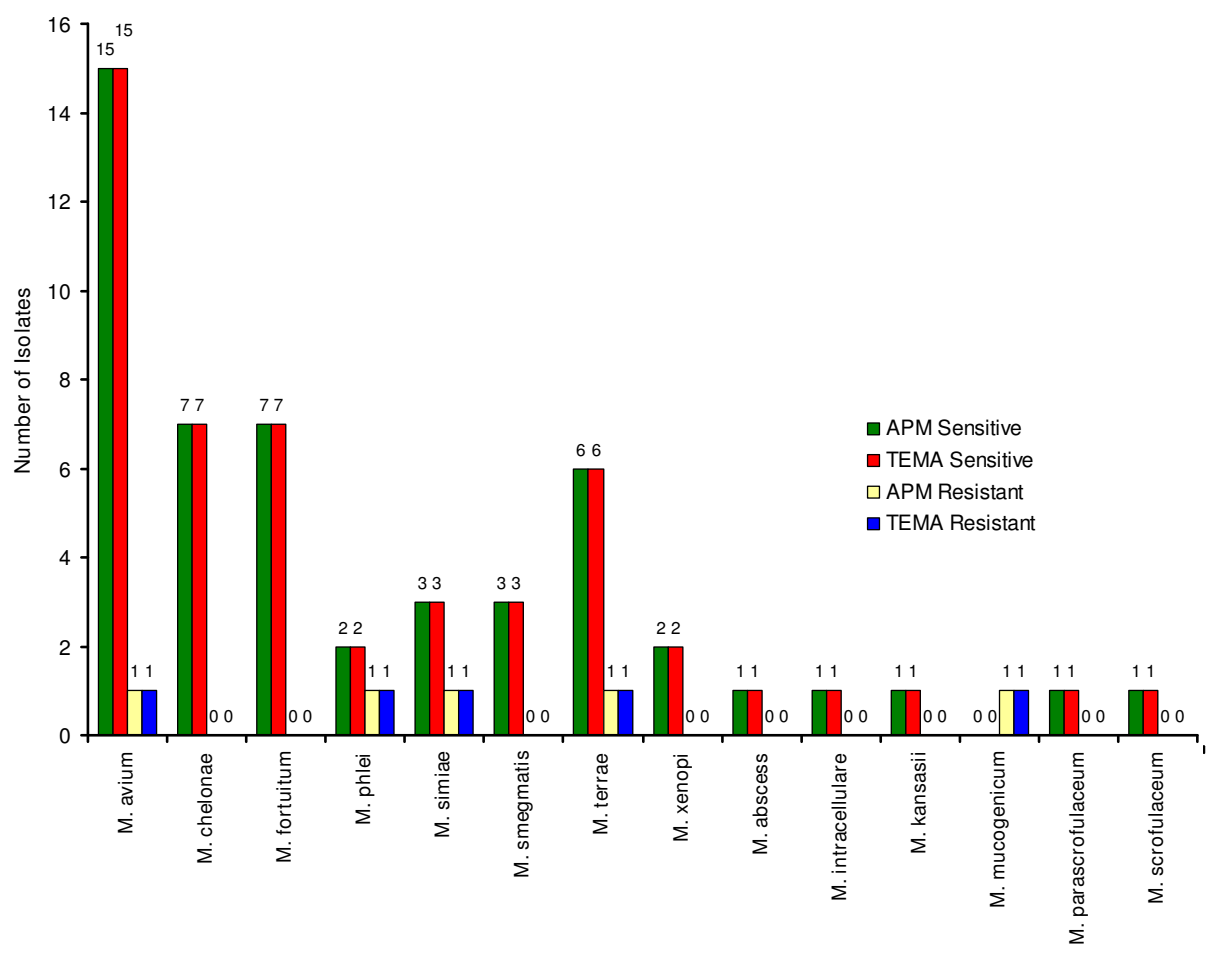

Figure 6

Drug resistance patterns in individual species of NTM against Ciprofloxacin. 


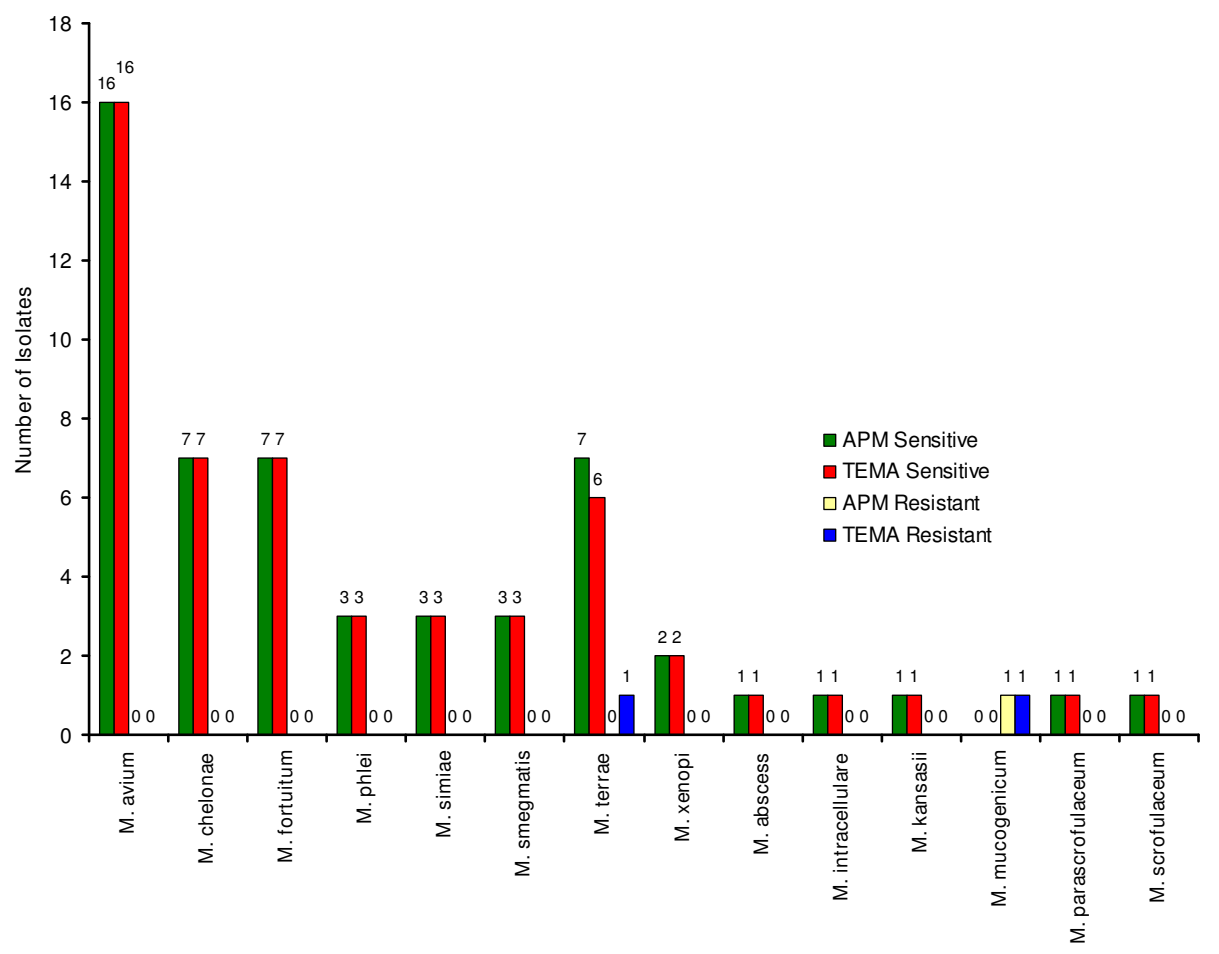

Figure 7

Drug resistance patterns in individual species of NTM against Ofloxacin.

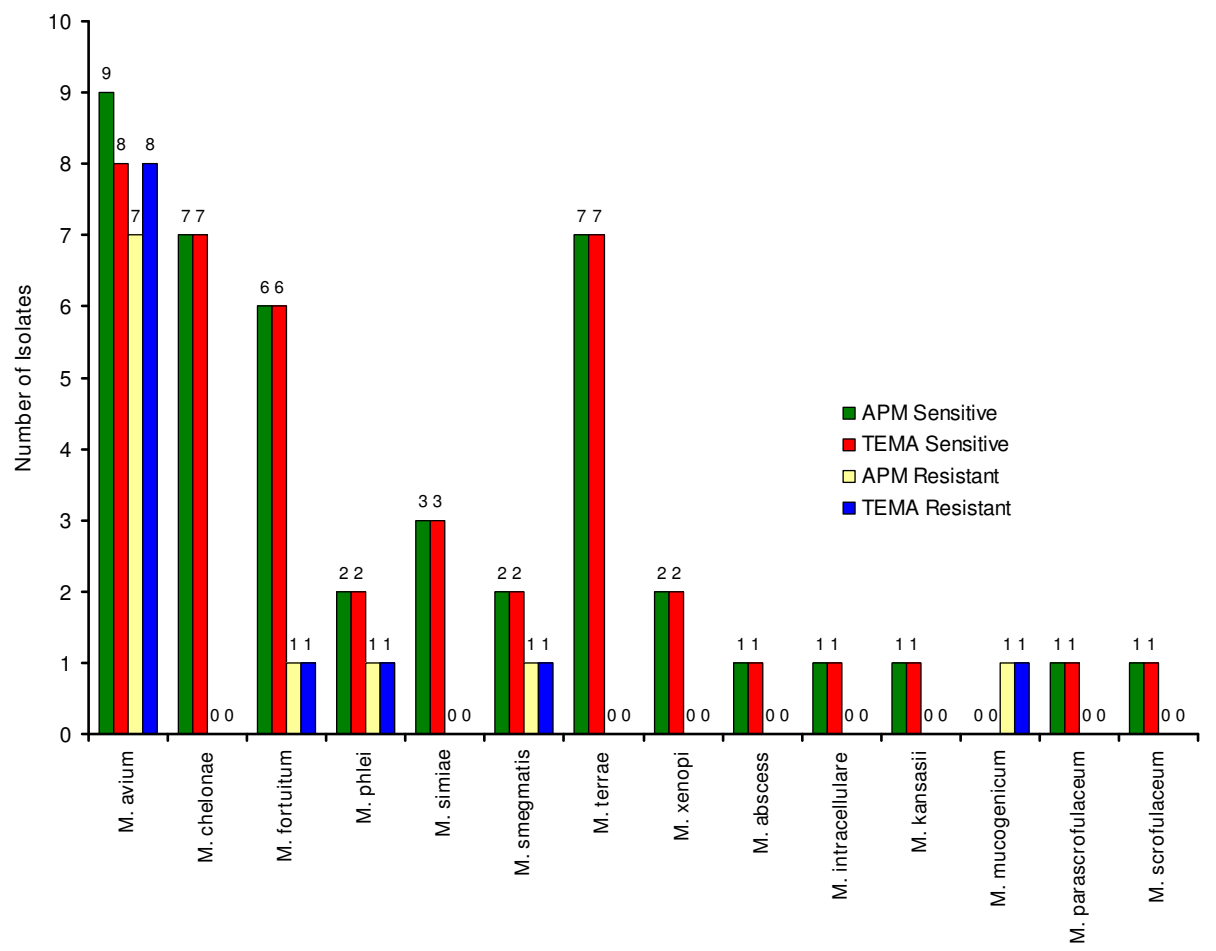

Figure 8

Drug resistance patterns in individual species of NTM against Azithromycin. 


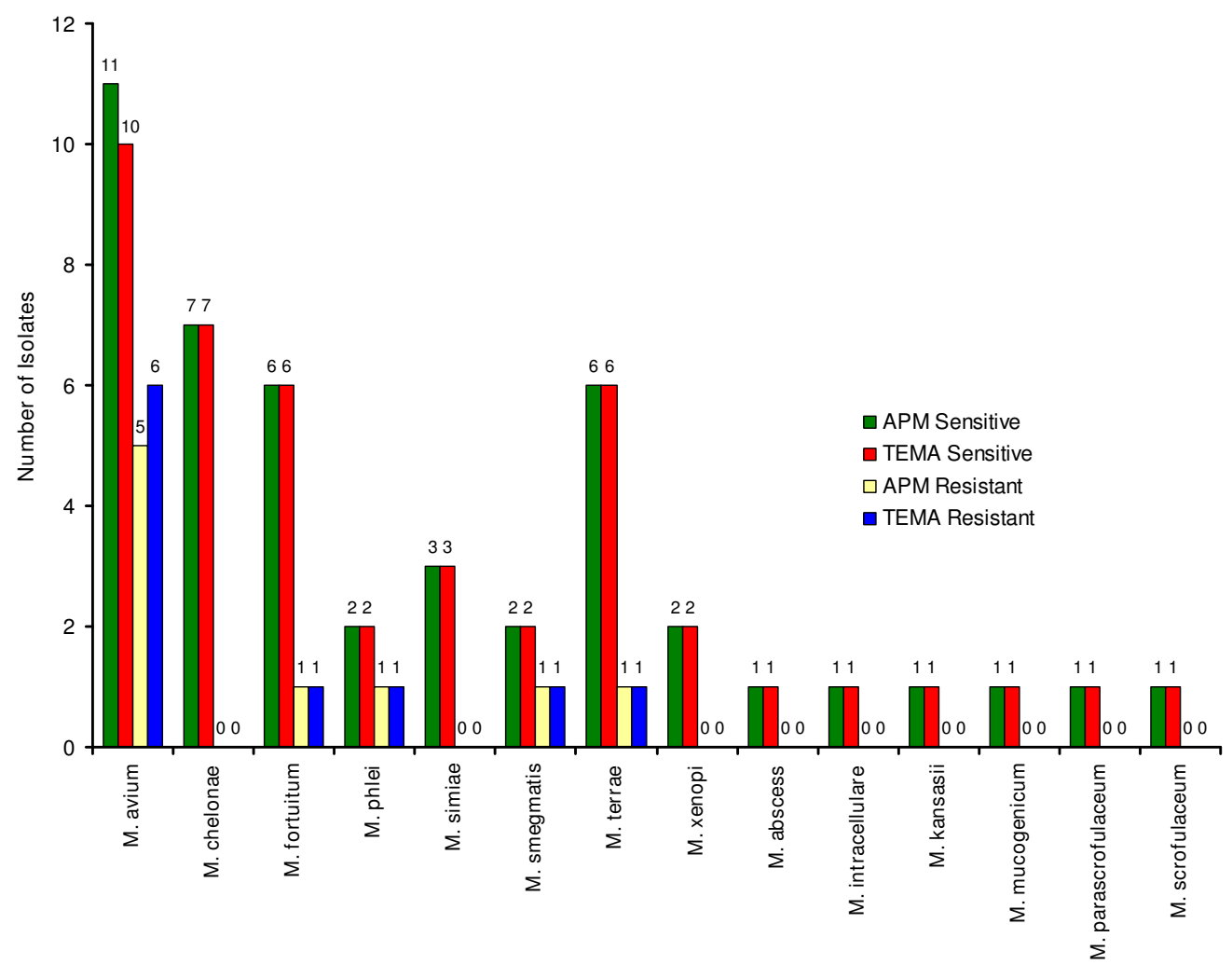

Figure 9

Drug resistance patterns in individual species of NTM against Clarithromycin.

obtained in a median time period of 5 to 9 days as compared to 12 to 25 days by APM.

\section{Discussion}

The epidemiology of mycobacterial infections had changed drastically in the past few decades and in-turn changing the treatment regimen and management practices, especially after AIDS epidemic [18]. In the terminal stages of AIDS, where $\mathrm{CD}_{4}^{+}$lymphocyte counts falls below 100, not only M. tuberculosis but several NTM also cause infection and can be isolated from blood, tissue, sputum and fecal samples [19]. The NTM disease not necessarily remains localized, but cause disseminated infection such as M. avium-intracellulare complex (MAC) [20]. The prognosis for the MAC infected AIDS patient is usually poor, largely due to the inherent resistance of these organisms to most of the available anti-tubercular drugs and have high toxicity.

Seventeen $(30.9 \%)$ of the 55 NTM isolates studied here were isolated from patients who were earlier treated with anti-tuberculosis therapy (ATT) without any clinical improvement. In clinical settings, these non-responsive cases are later labeled as Multi-drug resistant tuberculosis (MDR) by missing NTM infections. Similar observations were reported with other studies carried out in other countries such as Iran [21] and Brazil [22]. The clinical and radiological features of NTM infected patients like abnormal chest roentgenograms with infiltrations, nodular abscesses, cavities, lymphoadenopathy resembling TB can mislead the physician.

TEMA was effectively used to detect drug resistance in NTM isolates and was compared with the APM to which it had very good concordance (table 2 ). Previously similar results were obtained with $M$. tuberculosis (Data not published), prompted us to evaluate with other mycobacterial species. In this regard, the TEMA results were accurate, highly reproducible and rapid to determine the MICs of clinically significant mycobacteria.

However, before applying the TEMA as a routine drug susceptibility testing method, a multicentric and inter laboratory testing must be carried out with a supervision of international agencies such as World Health Organisation to validate our findings and to implement it in resource poor settings. We recommend that the TEMA, which is a calorimetric method, is more appropriate for level 3 reference laboratories to manipulate small volume of liquid cultures in a microplate format. 


\section{Competing interests}

The authors declare that they have no competing interests.

\section{Authors' contributions}

MMS participated in the collection and revival of strains, susceptibility testing, drafted the manuscript and performed the statistical analysis. KG participated in the design of the study, collection and characterization of strains. RS provided the clinical samples. SS conceptualized and designed the study. He also coordinated the study, critically evaluated the paper and arranged financial support. All authors have read and approved the final manuscript.

\section{References}

I. Horsburgh CR Jr, Selik R: The epidemiology of disseminated non-tubercular mycobacterial infection in the acquired immunodeficiency syndrome. Am Rev Respir Dis 1989, 139:4-7.

2. Nightingale SD, Byrd LT, Southern PM, Jockusch JD, Cal SX, Wynne BA: Incidence of Mycobacterium avium-intracellulare complex bacteremia in human immunodeficiency virus-positive patients. J Infect Dis 1992, 165(6): 1082-1085.

3. Contreras MA, Cheung OT, Sanders DE, Goldstein RS: Pulmonary infections with non-tubercular mycobacteria. Am Rev Respir Dis 1988, I37:149-152.

4. Collins FM: Mycobacterial disease, immuno suppression and acquired immunodeficiency syndrome. Clin Microbiol Rev 1989:360-377.

5. Philalay JS, Palermo CO, Hauge KA, Rustad TR, Cangelosi GA: Genes required for intrinsic multidrug resistance in Mycobacterium avium. Antimicrob Agents Chemother 2004, 48(9):34I2-8.

6. Nasha KA, Andini N, Zhang Y, Brown-Elliott BA, Wallace RJ Jr: Intrinsic macrolide resistance in rapidly growing mycobacteria. Antimicrob Agents Chemother 2006, 50(10):3476-8.

7. American Thoracic Society: Diagnosis and treatment of disease caused by Non-tubercular mycobacteria. Am J Respir Crit Care Med 1997, I 56: I-25.

8. Woodley CL, Kilburn JO: In vitro susceptibility of Mycobacterium avium and Mycobacterium tuberculosis strains to a sipropiperidyl rifampicin. Am Rev Respir Dis 1982, 1 26:586-587.

9. Heifets $L:$ MIC as a quantitative measurement of the susceptibility of Mycobacterium avium strains to seven antituberculosis drugs. Antimicrob Agents Chemother 1988, 32: I | 3 I-I I 36.

10. Steadham JE, Stall SK, Simmank JL: Use of the BACTEC system for drug susceptibility testing of Mycobacterium tuberculosis, M. kansasii, and M. avium complex. Diagn Microbiol Infect Dis 1985, 3:33-40.

II. Kuze F, Kurasawa T, Bando K, Lee $\mathrm{Y}$, Meakawa $\mathrm{N}$ : In vitro and in vivo susceptibility of atypical mycobacteria to various drugs. Rev Infect Dis 1981, 3:885-897.

12. Heifets LB, Iseman MD, Linhold-Levy PF, Kaes W: Determination of ansamycin MICs for Mycobacterium avium complex in liquid medium by radiometric and conventional methods. Antimicrob Agents Chemother 1985, 28:570-575.

13. Mshana RN, Tadesse G, Aabate G, Miörner H: Use of 3-(4, 5Dimethylthiazol-2-yl)-2, 5-Diphenyl TetrazoliumBromide for Rapid Detection of Rifampin-Resistant Mycobacterium tuberculosis. J Clin Microbiol 1998, 36(5):1214-1219.

14. Martin A, Morcillo N, Lemus D, Montoro E, da Silva Telles A, Simboli N, Pontino M, Porras A, León C, Velasco M, Chacon L, Barrera L, Rittaco V, Porteals F, Palomino JC: Multicentric study of MTT and resazurin assay for testing susceptibility to first line antituberculosis drugs. Int J Tuberc Lung Dis 2005, 9(8):90I-0.

15. Kent TP, Kubica PG: Public Health Mycobacteriology. A guide for the Level III Laboratory. Centers for Disease Control, Atlanta, Georgia; 1985.

16. Singh S, Gopinath K, Shahdad S, Kaur M, Singh B, Sharma P: Nontubercular mycobacterial infections in Indian AIDS patients detected by a novel set of ESAT-6 polymerase chain reaction primers. Jpn J Infect Dis 2007, 60(I): 14-8.
17. Heifets LB: Drug Susceptibility in the chemotherapy of Mycobacterial infections CRC press, Boca Raton, FL, USA; 1991: 100.

18. Subcommittee of the Joint Tuberculosis Committee of the British Thoracic Society: Management of opportunistic mycobacterial infections: Joint Tuberculosis Committee guidelines 1999. Thorax 2000, 55:210-18.

19. Gopinath K, Kumar S, Singh S: Prevalence of mycobacteremia in Indian HIV-infected patients detected by automated MB/ BacT automated culture system. Eur J Clin Microbiol Infect Dis 2008, 27:423-31.

20. Gopinath K, Kumar S, Sankar MM, Singh S: Novel Method for clearing red Blood Cell Debris from BacT/Alert ${ }^{\circledR}$ blood culture medium for improved microscopic and antimycobacterial drug susceptibility test results. J Clin Lab Anal 2007, 2I(4):220-6.

21. Bahrmand AR, Madani H, Samar G, Khalilzadeh L, Bakayev VV, Yaghli $M$, Babaei $M H$ : Detection and identification of non-tuberculous mycobacterial infections in 6,472 tuberculosis suspected patients. Scand J Infect Dis 1996, 28(3):275-8.

22. Matos ED, Santana MA, de Santana MC, Mamede P, de Lira Bezerra B, Panão DE, CSS Filho, ACM Lemos: Non-tuberculosis Mycobacteria at a Multi resistant Tuberculosis Reference Center in Bahia: Clinical Epidemiological Aspects. The Brazilian Journal of Infectious Diseases 2004, 8(4):296-304.
Publish with Biomed Central and every scientist can read your work free of charge

"BioMed Central will be the most significant development for disseminating the results of biomedical research in our lifetime. "

Sir Paul Nurse, Cancer Research UK

Your research papers will be:

- available free of charge to the entire biomedical community

- peer reviewed and published immediately upon acceptance

- cited in PubMed and archived on PubMed Central

- yours - you keep the copyright

Submit your manuscript here:

http://www.biomedcentral.com/info/publishing_adv.asp
BiolMedcentral 